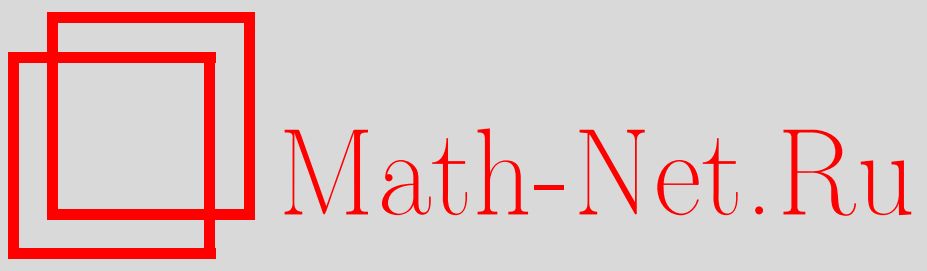

Ю. В. Туровский, В. С. Шульман, Радикалы в банаховых алгебрах и некоторые задачи теории радикальных банаховых алгебр, Функи. анализ и его прил., 2001, том 35, выпуск 4, 88-91

DOI: https://doi.org/10.4213/faa277

Использование Общероссийского математического портала MathNet.Ru подразумевает, что вы прочитали и согласны с пользовательским соглашением

http://www . mathnet.ru/rus/agreement

Параметры загрузки:

IP : 34.229 .45 .116

26 апреля 2023 г., 17:45:52

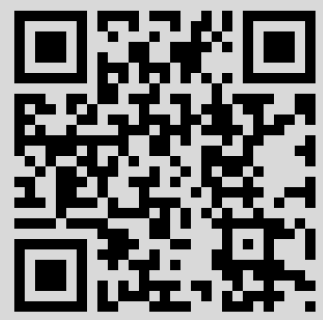


УДК 517.4

\title{
Радикалы в банаховых алгебрах и некоторые задачи теории радикальных банаховых алгебр
}

\author{
(c) 2001. Ю. В. Туровский, В. С. ШульмАН
}

1. Введение. Основы теории топологических радикалов заложены Диксоном [1]. Рассмотрим ее приложения к некоторым проблемам теории радикальных банаховых алгебр. Класс банаховых алгебр обозначим через $\{Б А\}$, его подкласс радикальных алгебр (т.е. таких алгебр $A$, которые совпадают со своим радикалом Джекобсона $\operatorname{rad} A)-$ через $\{$ РБА $\}$. Банахова алгебра $A$ называется рассеянной, если все ее элементы имеют (не более чем) счетные спектры, и компактной, если все ее элементы компактны (т.е. компактны все операторы $x \rightarrow a x a$ на $A$ ). Образ подмножества $M \subset A$ в факторалгебре $A / I$ по идеалу $I$ обозначим через $M / I$. Через $\rho(M)$ обозначается спектральный радиус ограниченного подмножества $M \subset A$, т. е. $\lim \left\|M^{n}\right\|^{1 / n}$, где $M^{n}-$ совокупность всех произведений по $n$ элементов из $M$, а норма множества определяется как супремум норм его элементов. Для семейства элементов из $A$, т. е. отображения $E: \Lambda \rightarrow A$, где $\Lambda-$ произвольное «индексное» множество, определяется также $l^{1}$-норма $\|E\|_{1}=\sum_{\alpha \in \Lambda}\|E(\alpha)\|$ (если она конечна, то семейство $E$ называется суммируемьмм и степени $E^{n}: \Lambda^{n} \rightarrow A, E^{n}\left(\alpha_{1}, \ldots, \alpha_{n}\right)=E\left(\alpha_{1}\right) \cdots E\left(\alpha_{n}\right)$, после чего $l^{1}$-спектральный радиус $\rho_{1}(E)$ вводится аналогично «обычному»: $\rho_{1}(E)=$ $\lim \left\|E^{n}\right\|_{1}^{1 / n}$. Разумеется, множество можно рассматривать как семейство, индексированное его же элементами, и вычислять $\rho_{1}(M)$ для суммируемых множеств $M \subset A$. Алгебра $A$ называется конечно (компактно) квазинильпотентной, если $\rho(M)=0$ для любого конечного (соответственно предкомпактного) множества $M \subset A$. Если $\rho_{1}(M)=0$ для всех суммируемых множеств $M$, то алгебру $A$ назовем 1-квазинильпотентной. Определим также $r(M)$ равенством $r(M)=\lim \sup _{n}\left(\sup \left\{\rho(a): a \in M^{n}\right\}\right)^{1 / n}$.

Одной из наиболее известных нерешенных задач теории алгебр из $\{$ РБА $\}$ является проблема существования нетривиального замкнутого идеала. Сильный результат здесь получен Войтыньским [2], который показал, что компактные радикальные алгебры имеют идеалы. В теореме 8 приведены дальнейшие утверждения о структуре идеалов таких алгебр.

Не решен и вопрос о радикальности проективного тензорного произведения алгебры из $\{\mathrm{PБA}\}$ на произвольную алгебру из $\{$ БА $\}$. В случае когда одна из алгебр коммутативна, решение дано в [3]. Связанный с этой задачей радикал строится в разд. 2 ; из результатов разд. 4 следует, что ответ положителен, если одна из алгебр компактна.

Неизвестно, является ли всякая алгебра из $\{$ РБА $\}$ компактно (или хотя бы конечно) квазинильпотентной. Соответствующий радикал строится в разд. 2 , а решение для компактного случая дается в разд. 4.

Еще недавно не было известно, является ли всякая алгебра из $\{$ РБА $\}$ топологически радикальной, т.е. не имеющей топологически неприводимых представлений в банаховых пространствах. Отрицательный ответ фактически получен 
Ридом в [4], где построен квазинильпотентный оператор без инвариантных подпространств. В теореме 8 утверждается, что и в классе компактных банаховых алгебр ответ отрицателен.

топологическим радикалом [1] называется отображение $A \rightarrow R(A)$, сопоставляющее любой банаховой алгебре ее замкнутый идеал и удовлетворяющее следующим условиям: 1) $R(R(A))=R(A)$; 2) $R(A / R(A))=0$; 3) $f(R(A)) \subset R(B)$, если $f: A \rightarrow B$ - непрерывный эпиморфизм; 4) если $I-$ замкнутый идеал в $A$, то $R(I)$ - идеал в $A$, содержащийся в $R(A)$ (если $R(I)=R(A) \cap I$, то $R$ называется наследственным). Алгебру $A$ называют $R$-радикальной, если $R(A)=A$, и $R$-коммутативной, если $A / R(A)$ коммутативна.

2. $\boldsymbol{\rho}$-радикалы. В этом разделе вводятся радикалы, реализующие «препятствия» в двух из перечисленных выше задач. Положим

$R_{\mathrm{cq}}(A)=\{a \in A: \rho(a M)=0$ для любого предкомпактного $M \subset A\}$,

$R_{\mathrm{sum}}(A)=\left\{a \in A: \rho_{1}(a M)=0\right.$ для любого суммируемого $\left.M \subset A\right\}$ (здесь $a M$ рассматривается как семейство, индексированное семейством $M)$. Нетрудно проверить, что $R_{\mathrm{cq}}(A) \subset R_{\text {sum }}(A)$.

ТЕОрема 1. (1) $R_{\mathrm{cq}}(A)-$ наибольший компактно квазинильпотентный идеал, $R_{\mathrm{sum}}(A)$ - наибольший 1-квазинильпотентный идеал.

(2) отображения $A \rightarrow R_{\mathrm{cq}}(A)$ u $A \rightarrow R_{\text {sum }}(A)$ - наследственные топологические радикаль.

(3) $\rho\left(M / R_{\mathrm{cq}}(A)\right)=\rho(M)$ для любого предкомпактного множества $M \subset A$, $\rho_{1}\left(M / R_{\text {sum }}(A)\right)=\rho_{1}(M)$ для любого суммируемого множества $M \subset A$.

Ясно, что алгебра $A$ компактно квазинильпотентна тогда и только тогда, когда $R_{\text {сq }}(A)=A$. Итак, $R_{\text {сq }}$ прямо связан с проблемой компактной квазинильпотентности радикальных алгебр. Сформулированная ниже теорема 2 связывает радикал $R_{\text {sum }}$ с задачей о радикальности тензорного произведения.

Teopema 2. $R_{\text {sum }}(A)=\{a \in A: a \otimes B \subset \operatorname{rad}(A \widehat{\otimes} B), B \in\{\mathrm{БA}\}\}$.

Алгебра $A$ называется совершенно радикальной, если $A \widehat{\otimes} B$ радикальна для всех $B \in\{$ БА $\}$, и совершенной, если $A \widehat{\otimes} B$ радикальна для всех $B \in\{$ РБА $\}$.

Следствие 3. (1) Алгебра А совершенно радикальна тогда и только тогда, когда она 1-квазинильпотентна.

(2) Любая компактно квазинильпотентная алгебра совершенно радикальна, а любая совершенно радикальная алгебра конечно квазинильпотентна.

\section{3. Рассеянный радикал.}

Теорема 4. (1) Любая банахова алгебра А имеет наибольший рассеянный udear $R_{\text {scat }}(A)$.

(2) $R_{\text {scat }}(A)=\{a \in A:$ спектр $\sigma(a b)$ не более чем счетен для любого $b \in A\}$.

(3) Отображение $A \rightarrow R_{\text {sсаt }}(A)$ - наследственный топологический ради$\kappa a \Omega$.

В частности, если $A \in\{$ БА $\}$ и $J-$ замкнутый идеал алгебры $A$, такой, что $J$ и $A / J$ рассеянны, то и $A$ рассеянна.

Теорема 5. Пространство примитивных идеалов (с топологией Джекобсона) сепарабельной рассеянной алгебры дискретно.

4. Гипокомпактный радикал. Напомним, что $a \in A$ компактен, если оператор $x \rightarrow a x a$ компактен. Компактные элементы алгебры $B(X)$ всех операторов, действующих в банаховом пространстве $X$, - это компактные операторы [5]. 
Будем называть алгебру гипокомпактной, если в любой ее ненулевой факторалгебре есть ненулевые компактные элементы. Примеры - расширения компактных алгебр посредством компактных (в том числе присоединение единицы к компактной алгебре).

Теорема 6. (1) Любая банахова алгебра А имеет наибольший гипокомпактный идеал $R_{\text {com }}(A)$.

(2) Отображение $A \rightarrow R_{\mathrm{com}}(A)$ - наследственный топологический ради$\kappa a \Omega$.

(3) $\rho(M)=\max \left\{\rho\left(M / R_{\mathrm{com}}(A)\right), r(M)\right\}$ для любого предкомпактного множества $M \subset A$.

(4) $R_{\text {com }}(A) \subset R_{\text {scat }}(A)$.

Пункт (3) теоремы 6 обобщает результат из [6] о равенстве $\rho(M)$ и $r(M)$ для ограниченных множеств операторов в конечномерном пространстве.

Теорема 7. (1) Всякая радикальная гипокомпактная алгебра компактно квазинильпотентна и как следствие совершенно радикальна.

(2) Всякая гипокомпактная алгебра совершенна.

Итак, на гипокомпактных алгебрах радикалы $R_{\mathrm{cq}}, R_{\mathrm{sum}}$ и $\mathrm{rad}$ совпадают. Но радикал $R_{T I}$ [1], равный пересечению ядер топологически неприводимых представлений, может быть у́же (п. (2)(i) теоремы 8).

ТЕорема 8. (1) Всякая бесконечномерная компактная радикальная алгебра имеет бесконечную иепь замкнутых идеалов.

(2) Существуют компактные алгебры $A_{1}, A_{2}, A_{3} \in\{P Б A\}$, такие, ито

(i) $A_{1}$ коммутативна, но не топологически радикальна,

(ii) $A_{2}$ не имеет максимальной (как цеепь подпространств) цепи замкнутьхх идеалов,

(iii) $A_{3}$ имеет бесконечномерный минимальный замкнутый идеал.

5. Ослабленная коммутативность. Коммутативные алгебры доставляют еще один пример класса алгебр, где радикалы $R_{\text {сq }}$ и $R_{\text {sum }}$ совпадают с радикалом Джекобсона. Это вытекает из следующих формул:

(1) $\rho(M)=\sup \{\rho(a): a \in M\}(M$ предкомпактно),

(2) $\rho_{1}(M) \leqslant \sum_{i \in \Lambda} \rho\left(a_{i}\right)\left(M=\left(a_{i}\right)_{i \in \Lambda}\right.$ суммируемо).

Эти свойства сохраняются для алгебр, выделяемых условиями ослабленной коммутативности в терминах теории алгебр Ли. Всякая ассоциативная алгебра - алгебра Ли с коммутатором $[a, b]=a b-b a$; в этом смысле мы будем говорить о подалгебрах Ли банаховых алгебр.

Теорема 9. Если банахова алгебра А порождена нильпотентной или конечномерной разрешимой подалгеброй Ли, то она $R_{\mathrm{cq}}$-коммутативна, $R_{\mathrm{cq}}(A)=$ $R_{\mathrm{sum}}(A)=\operatorname{rad}(A)$ и для любого предкомпактного (суммируемого) подмножества $M \subset A$ имеет место формула (1) (соответственно (2)).

Для приложений в теории спектра Тейлора [7] важен такой вопрос: является ли энгелевой банахова алгебра, порожденная нильпотентной (или, более общо, энгелевой) подалгеброй Ли? Мы называем нормированную алгебру Ли энгелевой, если на ней все операторы присоединенного представления ad $a: x \rightarrow[a, x]$ квазинильпотентны. Так же определяются энгелевы нормированные алгебры.

Теорема 10. Банахова алгебра $A$ rad-коммутативна тогда и только тогда, когда операторы аd $[a, b]$ квазинильпотентны для всех $a, b \in A$. В частности, всякая энгелева банахова алгебра rad-коммутативна. 
Следующие результаты частично отвечают на поставленный вопрос.

Теорема 11. (1) Рассеянная банахова алгебра, порожденная нильпотентной подалгеброй Ли, әнгелева.

(2) Гипокомпактная банахова алгебра, порожденная энгелевой подалгеброй Ли, энгелева.

\section{ЛИТЕРАТУРА}

1. Dixon P. Proc. London Math. Soc. (3), 74, 174-200 (1997). 2. Wojtynski W. Bull. Acad. Polon. Sci., Ser. Sci. math. astr. et phys., 26, No. 2, 109-113 (1978). 3. Aupetit B. Proprietes spectrales des algebres de Banach. Lect. Notes Math., Vol. 735, Springer-Verlag, Berlin, 1979. 4. Read C. J. J. London Math. Soc. (2), 56, 595-606 (1997). 5. Vala K. Ann. Acad. Sci. Fenn. Ser. A, I, 351, 1-8 (1964). 6. Berger M. A., Wang Y. Linear Algebra Appl., 166, 21-27 (1992). 7. Fainshtein A. S. J. Operator Theory, 29, 3-27 (1993).

Институт математики и механики АН Азербайджана e-mail: info@sinam.net

Вологодский политехнический институт, кафедра математики e-mail: victor@vstu.edu.ru
Поступило в редакцию 27 октября 2000 г. 